\title{
Theoretical and numerical analysis of wake vortices
}

\author{
D. SIPP, F. COPPENS \& L. JACQUIN
}

\author{
ONERA, \\ 29, avenue de la Division Leclerc \\ BP 72 \\ F-92322 CHATILLON CEDEX \\ e-mail : sipp@onera.fr
}

\begin{abstract}
The first part of the paper analyses the linear dynamics of a vortex pair thanks to a normal mode analysis. The basic flow is a superposition of two Lamb-Oseen vortices of radius $a$ and distance $b$. The long-wave Crow instability and the short-wave Widnall instabilities are fully characterized for different aspect ratios $a / b$ of the vortex pair. In particular, as $a / b$ increases, it is shown that the antisymmetric Widnall instabilities are favoured for short-wave perturbations. This constitutes an explanation for the results obtained by Leweke \& Williamson (1998). The second part of the paper deals with turbulence in the Batchelor vortex. A 3D Large Eddy Simulation (LES) shows that, starting from a linearly unstable wake-type vortex, high levels of turbulence can be obtained. But, the mean-flow then rapidly evolves towards a stable state. These results are in accordance with those of Ragab \& Sreedhar (1995).
\end{abstract}

\section{Introduction}

This paper analyses some linear and non-linear properties of the dynamics of vortices. Two problems will be discussed. The first concerns the linear dynamics of two Lamb-Oseen vortices. The second deals with the turbulence effects on a Batchelor-type vortex. These constitute two different aspects of the general airplane vortex wake hazard problem. Linear stability of two counterrotating vortices, including the (long-wave) Crow instability (Crow 1970) and the (short-wave) Widnall instability (Tsai \& Widnall 1976 and Moore \& Saffman 1975), may contribute to an aircraft vortex wake dispersion. In the present paper, these two instabilities are fully characterised in a unified frame by means of a normal mode analysis. The development of turbulence and its impact on aircraft vortices is still a matter of question. A temporal numerical simulation of an unstable Batchelor vortex is performed in order to get an insight into this problem.

The first part of this paper is devoted to linear dynamics. We first obtain the bending wave oscillation frequencies of a single Lamb-Oseen vortex by means of a shooting method. It is shown that a single Lamb-Oseen vortex is stable. Then, a spectral collocation and matrix eigenvalue method is used to analyse the dynamics of a vortex pair. In particular, the Crow instability in the long-wave limit and the Widnall instabilities are recovered by this general method. We also prove that increasing the core radius makes the interaction between the two vortices stronger. It is shown, for example, that the antisymmetric mode of the Widnall instability is promoted as the core radius increases.

The second part deals with turbulence in the Batchelor vortex. It is shown that high levels of turbulence can be obtained in the vortex. Starting from a linearly unstable wake-type vortex, the 
mean-flow evolves towards a stable state through an increase of its axial momentum. This is in accordance with the results of Ragab \& Sreedhar (1995).

\section{The linear dynamics of a vortex pair : a normal mode analysis}

The present section focuses on theoretical aspects concerning the linear temporal dynamics of vortices (see Sipp \& Jacquin 1998).

A variety of physical mechanisms exists when considering the dynamics of a single vortex. First if the magnitude of the circulation diminishes with increasing radius in some region of the flow then centrifugal instability should occur. Secondly, in the presence of an axial flow, the vortex is unstable (see Lessen, Singh \& Paillet 1974 and Mayer \& Powell 1992).

In this section, we focus on $2 \mathrm{D}$ vortices with outward increasing circulation leaving aside instabilities due to the axial flow as well as centrifugal type instabilities. A single Lamb-Oseen vortex fulfils these conditions and we prove that this vortex is indeed stable. Instability may arise through linear interactions between two vortices. In this section we will therefore study the linear dynamics of two counter-rotating Lamb-Oseen vortices. Let $\Gamma$ be the circulation and $a$ the core radius of each individual vortex and let $b$ be their mutual distance.

Note that this flow is not a stationary solution of the inviscid Euler equations, even in the frame moving with the vortices. In the case of a weakly strained vortex, Moore \& Saffman (1975) explained how the vortex adapts to the weak strain field. In the general case, the asymptotic stationary state can be determined numerically. However, the superposition of two counter-rotating Lamb-Oseen vortices is often used as an approximate model for representing a flow corresponding to vortex pairs in practical applications (see e.g. Leweke \& Williamson 1998). This choice leads to an analytical expression for the basic flow where the aspect ratio $a / b$ can easily be adjusted. It therefore constitutes a good compromise for our purpose.

The streamlines of the basic flow field are sketched in figure 1 for $a / b=0.2$. The cartesian components of velocity are :

$$
u=\psi_{y}, \quad v=-\psi_{x}, \quad w=0
$$

where $\psi(x, y)$ is the streamfunction and the subscripts $x$ and $y$ denote partial spatial derivatives.

Normal modes are sought in the form $e^{\sigma t} e^{i k z} \phi(x, y)$ where $\phi=u^{\prime}, v^{\prime}, w^{\prime}, p^{\prime}$ stands for the velocity and pressure perturbations. $k$ is the real $O z$ wavenumber and $\sigma$ is the complex amplification rate. By linearising the full incompressible viscous Navier-Stokes equations around the basic flow field, we obtain the non-parallel versions of the Orr-Sommerfeld and Squire equations :

$$
\left[(\sigma-\nu \Delta)\left(\begin{array}{cc}
0 & \Delta \\
\mathcal{V} & \mathcal{W}
\end{array}\right)-\left(\begin{array}{cc}
\mathcal{Q} & \mathcal{R} \\
\mathcal{S} & \mathcal{T}
\end{array}\right)\right]\left(\begin{array}{l}
u^{\prime} \\
v^{\prime}
\end{array}\right)=0
$$

with the following linear operators :

$$
\begin{aligned}
\Delta & =\partial_{x x}+\partial_{y y}-k^{2} \\
\mathcal{V} & =\partial_{x x}-k^{2} \\
\mathcal{W} & =\partial_{x y} \\
\mathcal{Q} & =\psi_{x x}\left(\partial_{x x}-\partial_{y y}-k^{2}\right)+2 \psi_{x y} \partial_{x y}-2 \zeta_{x} \partial_{x}-\zeta_{x x}
\end{aligned}
$$

ESAIM: Proc., Vol. 7, 1998, $398-407$ 


$$
\begin{aligned}
\mathcal{R} & =-\left(\psi_{y} \partial_{x}-\psi_{x} \partial_{y}-\psi_{x y}\right) \Delta-2 \psi_{x y} \partial_{x x}+2 \psi_{x x} \partial_{x y}-\zeta_{x} \partial_{y}-\zeta_{y} \partial_{x}-\zeta_{x y} \\
\mathcal{S} & =-\left(\psi_{y} \partial_{x}-\psi_{x} \partial_{y}+\psi_{x y}\right)\left(\partial_{x x}-k^{2}\right)+\psi_{x x} \partial_{x y} \\
\mathcal{T} & =-\left(\psi_{y} \partial_{x}-\psi_{x} \partial_{y}+\psi_{x y}\right) \partial_{x y}+\psi_{x x}\left(\partial_{y y}+k^{2}\right)-k^{2} \zeta
\end{aligned}
$$

In these expressions, $\partial_{x y}=\partial^{2} / \partial x \partial y$, etc and $\zeta$ is the $O z$ vorticity of the basic flow field : $\zeta=$ $-\left(\psi_{x x}+\psi_{y y}\right) . \nu$ denotes the kinematic viscosity.

The symmetries of the basic flow field enable us to prove that the eigenmodes may be decomposed into two independent subsets of symmetric $\left(u^{\prime}(x, y)\right.$ even and $v^{\prime}(x, y)$ odd with respect to the $y$ variable) and antisymmetric modes (vice and versa).

The amplification rate $\sigma$ is a function of the radius $a$, the distance $b$, the viscosity $\nu$, the circulation $\Gamma$ and the wavenumber $k$. On dimensional grounds, we expect :

$$
\sigma \frac{2 \pi b^{2}}{\Gamma}=f\left(\frac{a}{b}, k a, \frac{\Gamma}{\nu}\right)
$$

Studying the linear dynamics of the basic flow is equivalent to determining the values of this complex function for all eigenmodes. This will be achieved with a 2D spectral Gauss collocation method. The various parameters $k a, a / b$ and $R e$ can be set arbitrarily and an eigenmode can be continuously followed when varying these parameters. For asymptotic values of the parameters, results may be compared to those obtained with more simple flows and/or with other suitable techniques. Three cases are considered.

- Firstly, in the long-wave limit $(k a \rightarrow 0)$, Crow (1970) proved that the mutual induction of a pair of infinite line vortices of opposite sign makes them unstable to coupled sinusoidal disturbances. A filament vortex method was used for this purpose. This provides the asymptotic values $f(k a \rightarrow 0, a / b \rightarrow 0, R e=\infty)$ of some eigenmodes. The instability mechanism has been confirmed by numerous observations and simulated by several authors.

- Secondly, Moore \& Saffman (1975) and Tsai \& Widnall (1976) showed that a strained vortex is unstable to short-wave instabilities ( $k a$ of order 1$)$. The interaction of Kelvin waves with a weak strain field may be studied by means of a multiple time scale analysis. A precise knowledge of the dynamics of a single Lamb-Oseen vortex is first required. This was achieved by means of a shooting method which determines the spatial structure and eigenfrequencies of the Kelvin waves. The whole process gives the asymptotic values $f(k a, a / b \rightarrow 0, R e=\infty)$ of the eigenmodes whose wavenumber $k a$ is of order 1 . In the case of instability, the mechanism corresponds to a triadic resonance between the strain field and two Kelvin waves of the same frequency, the same axial wavenumber $k a$ and azimuthal wavenumber $m$ and $m+2$. A similar short-wave instability can be found in other complex flows like in flattened two-dimensional Taylor-Green vortices (see Sipp \& Jacquin 1998).

These numerical results are summarised in figure 2 and 3 . The oscillation frequencies $\omega$ of the bending waves $\left(e^{i \omega t} e^{i k z} e^{i \theta} \phi^{\prime}(r)\right)$ have been sketched in figure 2 . These waves are stable. In figure 3 we have plot the amplification rates concerning the Crow and the Widnall instabilities. The Crow instability only affects the symmetric mode and is due to the bending wave labelled 0 . The 3 remaining unstable bands - the Widnall instabilites -, due to resonances of the bending waves labelled 1, 2 and 3, affect symmetric and antisymmetric modes with the same strength. 
In summary, calculations will be conducted to verify the agreement between the results given in each of these asymptotic cases and those given by a complete normal mode analysis of two vortices. The validity domain in $k a$ and $a / b$ will be defined in each case.

\subsection{Global results}

A 2-D spectral Chebyshev-Gauss Collocation method, associated with an exponential or an algebraic mapping, was used to discretise the remaining spatial derivatives $\frac{\partial}{\partial x^{n}}$ and $\frac{\partial}{\partial y^{m}}$ of equation (2). We are then lead to a generalised eigenvalue problem, $A w=\sigma B w$, where $A$ and $B$ denote two matrices and $(\sigma, w)$ is an eigenvalue/eigenvector. This problem is solved with LAPACK routines on a C90 CRAY.

Calculations have been conducted in the case $a / b=0.2$ and $R e=\infty$. We use 34 Chebyshev polynomials in the $x$ direction and 76 in the $y$ direction. We place the collocation points in the rectangle $-5 \leq x / a \leq 5,-7.5 \leq y / a \leq 7.5$ with an exponential or algebraic mapping. The two vortices are located at $(x / a=0, y / a= \pm 2.5)$.

First we consider the case $k a=5$. Two calculations have been conducted in order to get the symmetric and the antisymmetric modes. They enabled us to identify the bending waves labelled 0 to 2 (see figure 2 for definition). We then follow these modes for $k a$ decreasing from 5 to 0 with a step of $\Delta(k a)=-0.05$ or -0.1 . Results are given in figure 4 . In each plot we have sketched the imaginary part of the amplification rate $\sigma 2 \pi a^{2} / \Gamma$ versus the vertical wavenumber $k a$. Results given by the shooting method for one Lamb-Oseen vortex are given by the solid line whereas the squares and the filled triangles represent the calculated antisymmetric and symmetric mode eigenfrequencies.

In this figure, when $k a$ is of order 1 , the symmetric and antisymmetric modes exhibit the same eigenfrequency. This means that the dynamics of the two vortices are independent. The bending waves (figure 4) follow the dynamics of a single vortex, except for certain values of $k a$, outlined by circles. These regions which coincide with the unstable bands of figure 3 , will be studied in detail in the following.

\subsection{The Widnall instability}

In the left plot of figure 5, we give the results for the bending wave labelled 1 , for $1.5 \leq k a \leq 3.1$. We have sketched the amplification rate $\sigma_{r} 2 \pi b^{2} / \Gamma$ and $\sigma_{i} 2 \pi a^{2} / \Gamma$ versus the wavenumber $k a$. The solid and the dashed line represent results of the asymptotic stability analysis (see Moore \& Saffman 1975). The circles and the squares correspond to antisymmetric mode results and the filled triangles to symmetric ones. The agreement between the different calculations is very good : both the width of the unstable band and the maximum amplification rate are well recovered with the 2D Chebyshev calculation. Two oscillatory eigenvalues are colliding giving rise to a non-oscillating instability. The right plot of figure 5 shows the symmetric (or antisymmetric) eigenmode $u^{\prime}(x, y)$ and $v^{\prime}(x, y)$ in the half plane $y>0$, for $k a=2.5$. This picture is analogous to that of elliptic instability eigenmodes obtained in stretched Taylor-Green vortices (see Sipp \& Jacquin 1998) and to that obtained in unbounded flows (the vertical vorticity forms a dipole whose axis is aligned with the stretching direction, see Waleffe 1990). 


\subsection{The Crow instability}

The long-wave limit is analysed in figure 6 . In this case, the perturbation fields on the two vortices interact so that a different behaviour is expected for symmetric and antisymmetric modes. The solid line corresponds to a single vortex. Dotted, dashed and long-dashed lines refer to the results of the filament vortex method (see Crow 1970). This plot shows that when $k a$ is large, the antisymmetric and symmetric modes concentrate along the one vortex curve (solid line). This is in accordance with the decrease of the interaction between the perturbation fields when $k a$ increases. But when $k a$ decreases the frequencies defer and the symmetric and antisymmetric modes collapse with the curves predicted by Crow's filament vortex method. The antisymmetric and symmetric modes could be followed down to $k a=0.15$ and $k a=0.30$ respectively. Below these values, the numerical code delivers several possible modes with different eigenvalues.

\subsection{Influence of the aspect ratio $a / b$ on the Widnall instability occurring for the bending wave labelled 1}

In this section, we analyse the influence of the aspect-ratio $a / b$ on the Widnall instability occurring for the bending wave labelled 1 . The left plot of figure 7 shows the evolution with the aspect ratio $a / b$ of the characteristics of the unstable band : the upper and lower bounds $k_{1} a$ and $k_{2} a$ and the locus of maximum instability $k_{m} a$ are given. In the right plot, we sketched the corresponding maximum amplification rate $\sigma_{m} 2 \pi b^{2} / \Gamma$. These results are compared with those of the asymptotic analysis (solid line). The two approaches are in good agreement for small aspect ratios $a / b$. But these differ as $a / b$ increases : we observe that the antisymmetric mode is favoured. This is in agreement with the experiments conducted by Leweke \& Williamson (1998) who found that long-wave instabilities only affect the symmetric mode whereas the short-wave perturbations are antisymmetric. Consequently, the mutual induction between the perturbation fields on the two vortices leads to a promotion of the antisymmetric mode.

\section{On the development of turbulence in vortices : a numerical simulation}

In the near-field of an aircraft wake, the trailing vortex is three-dimensional, composed of a tangential velocity and an axial velocity deficit. The vortex is described here with the commonly used Batchelor similarity model, also referred to as the 'q-vortex'. The initial velocity distribution is given by:

$$
\begin{aligned}
& u_{\theta}=\frac{q}{r}\left(1-e^{-r^{2}}\right) \\
& u_{z}=1-e^{-r^{2}}
\end{aligned}
$$

where $q$ is proportional to the ratio of maximum tangential velocity to axial velocity deficit. Mayer and Powell have conducted extensive studies of the linear stability response of this vortex to helical perturbations (Kelvin waves). They indicate that $q$ is a determining parameter of instability and that the vortex is generally stabilized for values $q>1.5$. Here a value of $q=1$ is chosen and the vortex is submitted to a helical eigenmode of the form $\phi(r, \theta) e^{i m \theta+k z}$ computed by Ragab. 
The choice of wavenumbers is $m=1$ and $k=0.7$, which correspond to one of the most amplified modes, with a theoretical amplification rate $\sigma=0.13$.

The simulation was carried out with the ONERA Pegase incompressible large-eddy simulation code, at a Reynolds number of 36000 based on initial core radius and maximum tangential velocity. Spatial schemes are second-order for convection and fourth-order for diffusion and time integration is second-order. The subgrid-scale model, known as 'selective mixed-scale', is a dynamic Oneradeveloped improvement of the Smagorinsky formulation. Computations were run on a Cray C90 computer on a $177^{2} \times 51$ orthogonal grid.

Figure 8 shows the turbulence rate $\frac{\sqrt{K}}{V_{\infty}}$ where $K$ denotes the kinetic energy of the fluctuating velocity field averaged over the calculation box. After a phase of linear amplification, in accordance with theory, we observe a non-linear saturation. The snapshots of the vorticity fields and detailed analysis of the data statistics confirm the presence of fully developed turbulence (see Coppens 1998). Concerning the mean-field, figure 8 shows a reduction of the axial velocity deficit, while the tangential velocity remains mostly unaffected. This shows that, despite a large turbulence level, one does not observe any significant radial diffusion of the angular momentum. As a consequence, the factor $q$ increases so that the vortex evolves towards a linearly stable state. The same results were obtained by Ragab \& Sreedhar (1995) with a similar simulation (based on different numerical schemes and models). This sustains the expectation that the far-field aircraft vortex wakes behave as in a laminar regime (see Zeman 1995) apart from the vortex pair instabilities discussed in the first part.

\section{References}

1 Batchelor, G.K.: Axial flow in traling line vortices. J. Fluid Mech. 20 (1964), 645-658.

2 Coppens, F.: Simulations numériques sur le développement de la turbulence dans un tourbillon. Thèse de l'Institut Polytechnique de Toulouse (1998).

3 Crow, S.C.: Stability theory of a pair of trailing vortices. AIAA J. 8 (1970), 2172-2179.

4 Moore, D.W. \& Saffman, P.G.: The instability of a straight vortex filament in a strain field. Proc. R. Soc. Lond. A 346 (1975), 413-425.

5 Lessen, M., Singh, P.J. \& Paillet, F. The stability of a trailing line vortex. Part 1. Inviscid theory. J. Fluid Mech. 360 (1974), 753-763.

6 Leweke, T. \& Williamson, C.H.K. Cooperative elliptic instability of a vortex pair. J. Fluid Mech. 360 (1998), 85-119.

7 MAYer, E.W. \& Powell, K.G.: Viscous amd inviscid instabilities of a trailing vortex. J. Fluid Mech. 245 (1992), 91-114.

8 Moore, D.W. \& Saffman, P.G.: The instability of a straight vortex filament in a strain field. Proc. R. Soc. Lond. A 346 (1975), 413-425.

9 RAGAB, S.: private communication (1995).

10 Ragab,S. \& SReedhar, M.: Numerical simulation of vortices with axial velocity deficits. Phys. Fluids 7 (1995), 549-558.

11 SipP, D. \& JACQuin, L.: Linear dynamics of two counter-rotating Lamb-Oseen vortices. Submitted to the J. Fluid Mech. (1998)

12 SipP, D. \& JACQuin, L.: Elliptic instability in 2D flattened Taylor-Green vortices. Phys. Fluids 10 (1998), 839-849.

13 Tsai, C.Y. \& Widnald, S.E.: The instability of short waves on a straight vortex filament in a weak externally imposed strain field. J. Fluid Mech. 73 (1976), 721-733.

14 Waleffe, F.: On the three-dimensional instability of strained vortices. Phys. Fluids A 2 (1990), 76.

15 Zeman, O.: The persistence of trailing vortices: A modeling study. Phys. Fluids 7, 135. 


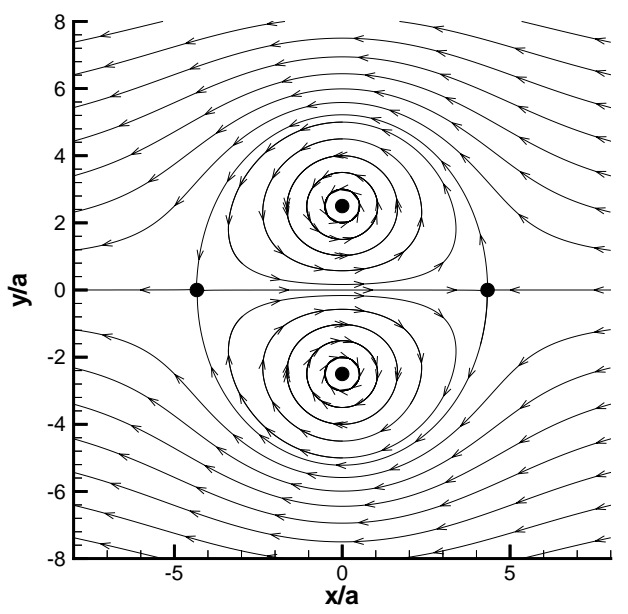

Figure 1: Streamlines of the basic flow field constituded of two counter-rotating Lamb-Oseen vortices, in the frame moving with the vortices. Case $a / b=0.2$.

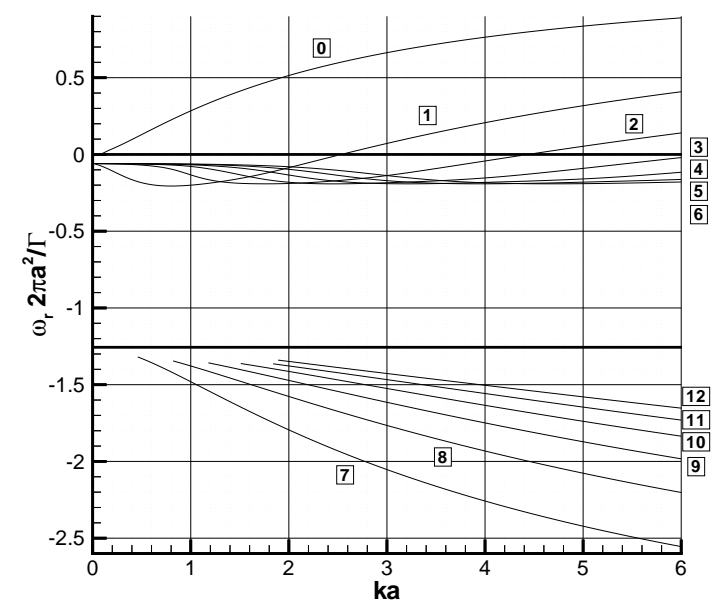

Figure 2: Bending mode oscillation frequencies of a Lamb-Oseen vortex $(\Gamma, a)$ obtained by means of a shooting method. 


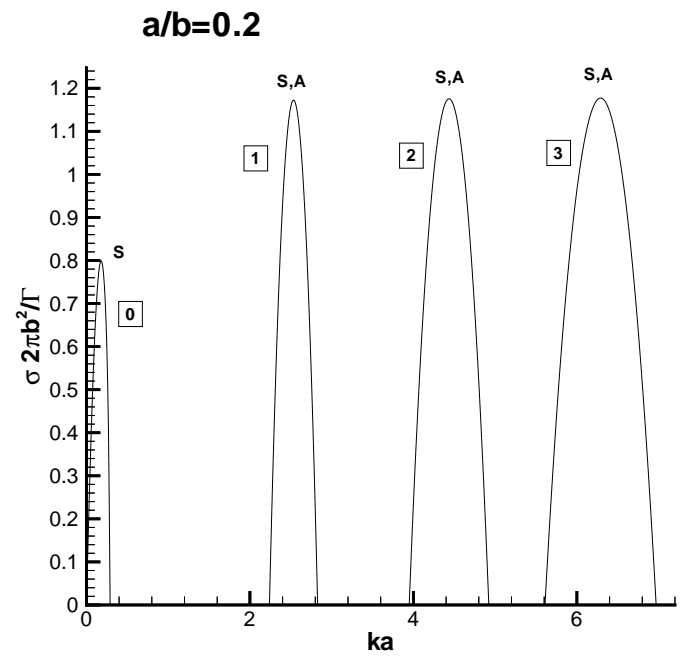

Figure 3: Crow and Widnall instabilities in the case $a / b=0.2$. The symbols $\mathrm{S}$ and A refer to symmetric and antisymmetric modes.

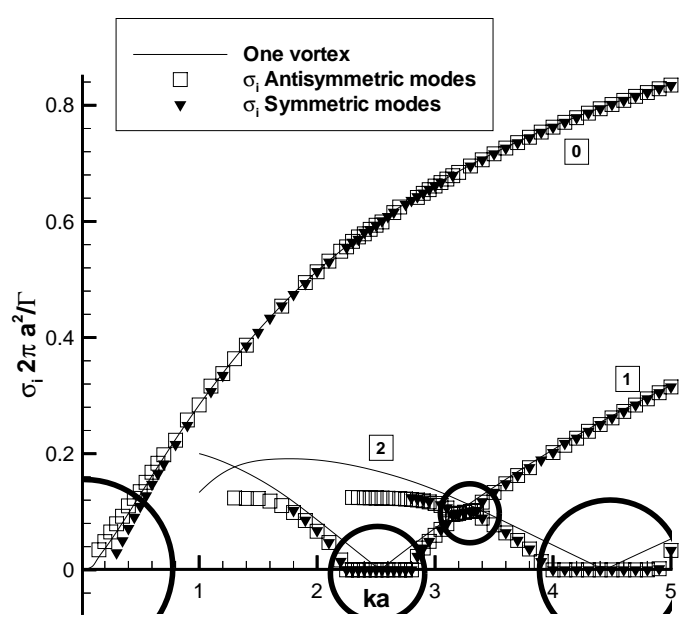

Figure 4: Comparison between the bending mode eigenfrequencies given by the shooting method for one vortex (lines) and those given by a complete normal mode analysis of a vortex pair (symbols) with $a / b=0.2$. 


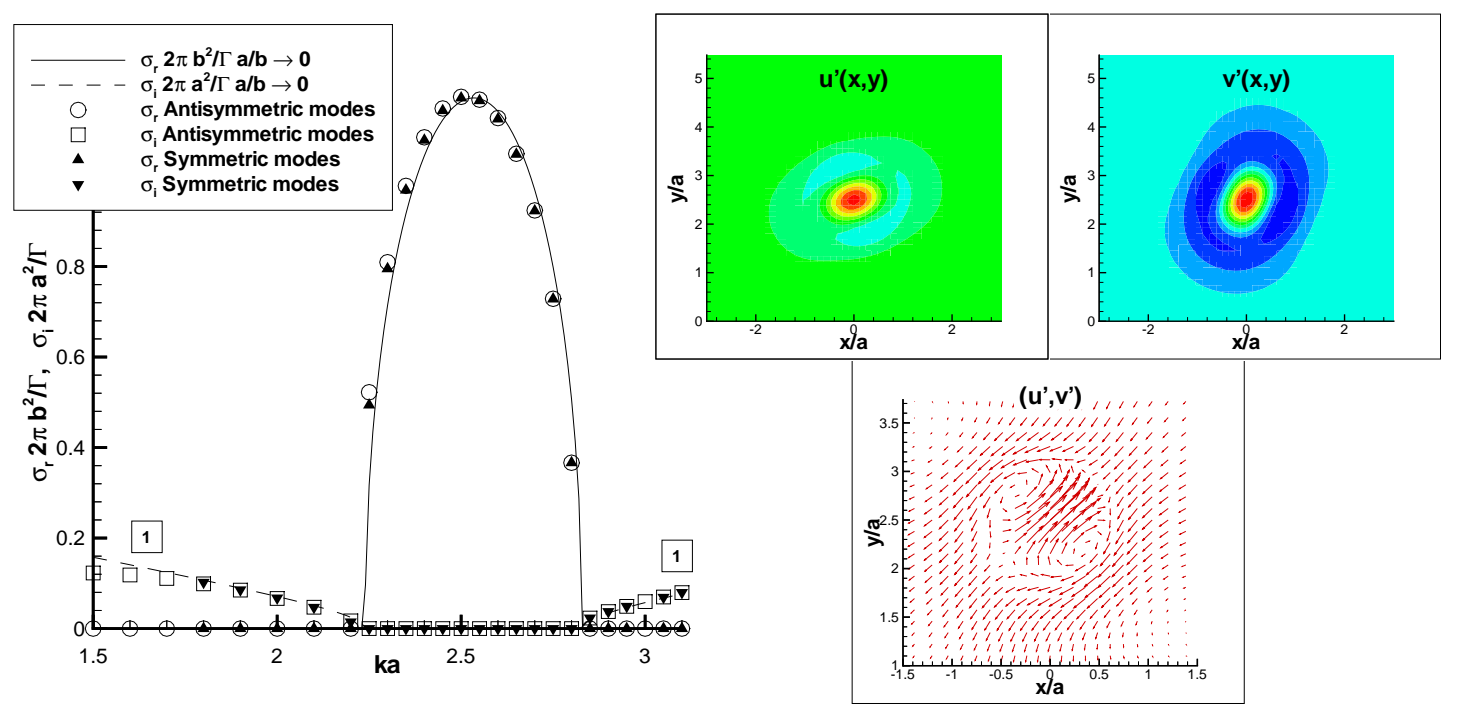

Figure 5: Widnall instability occurring for the bending wave labelled 1. Left plot : comparison between results given by the asymptotic stability analysis (lines) and those given by the complete normal mode analysis of a vortex pair (symbols). Right plot : unstable eigenvector obtained for $k a=2.5$.

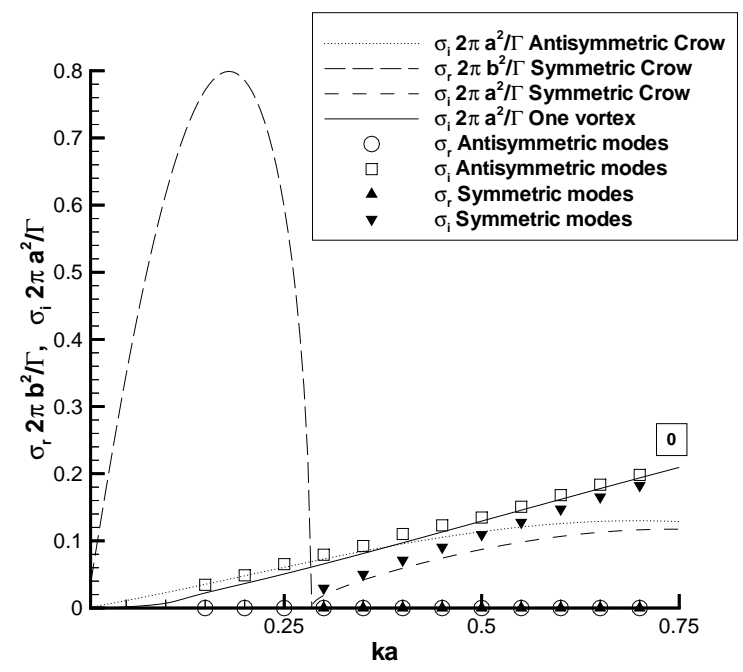

Figure 6: The long-wave limit : the bending wave labelled 0 (slow branch). The dotted and dashed lines are results given by the filament vortex method whereas the solid line are results obtained with the shooting method for a single vortex. Symbols represent results given by the normal mode analysis of a vortex pair. 

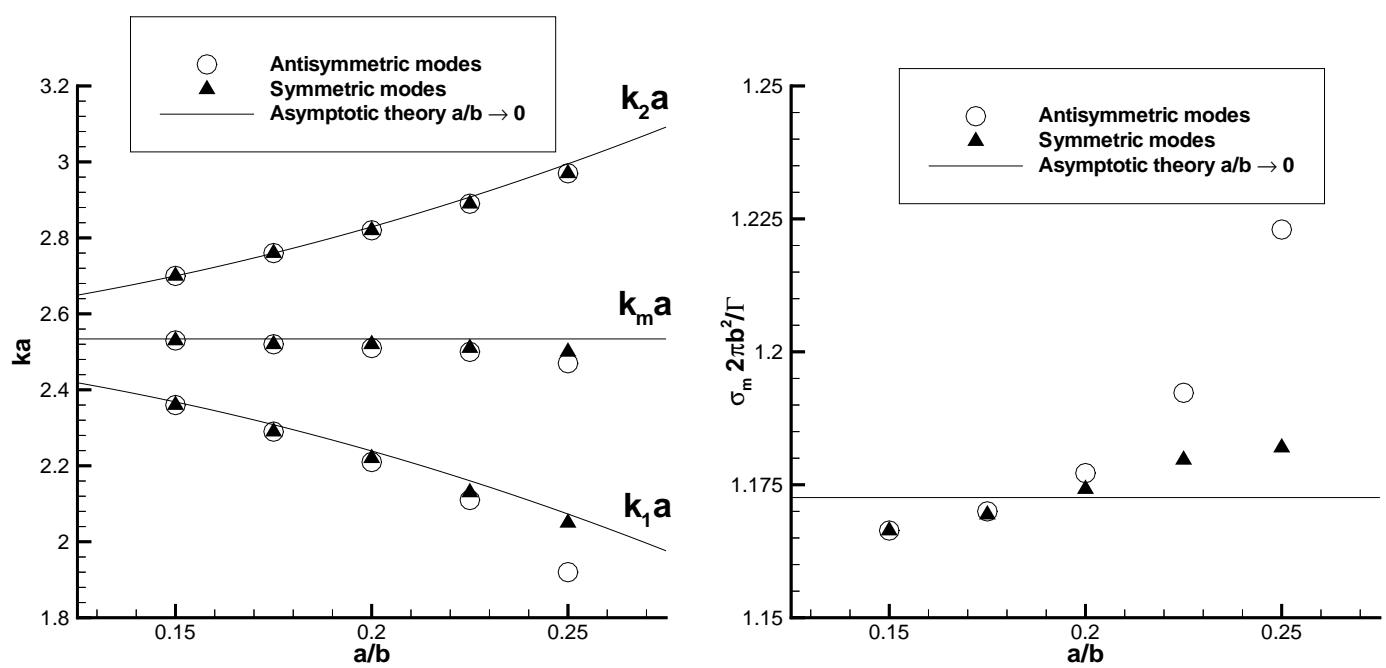

Figure 7: Behaviour of the Widnall instability for the bending wave labelled 1, for different values of the aspect ratio $a / b$. Left plot : the unstable band is located in the range $k_{1} a \leq k a \leq k_{2} a$ and maximum instability occurs for the wavenumber $k_{m} a$. Right plot : maximum amplification rate $\sigma_{m} 2 \pi b^{2} / \Gamma$ corresponding to the wavenumber $k_{m} a$. In all plots, the lines correspond to results given by the asymptotic stability analysis and the symbols refer to the normal mode analysis of a vortex pair. 

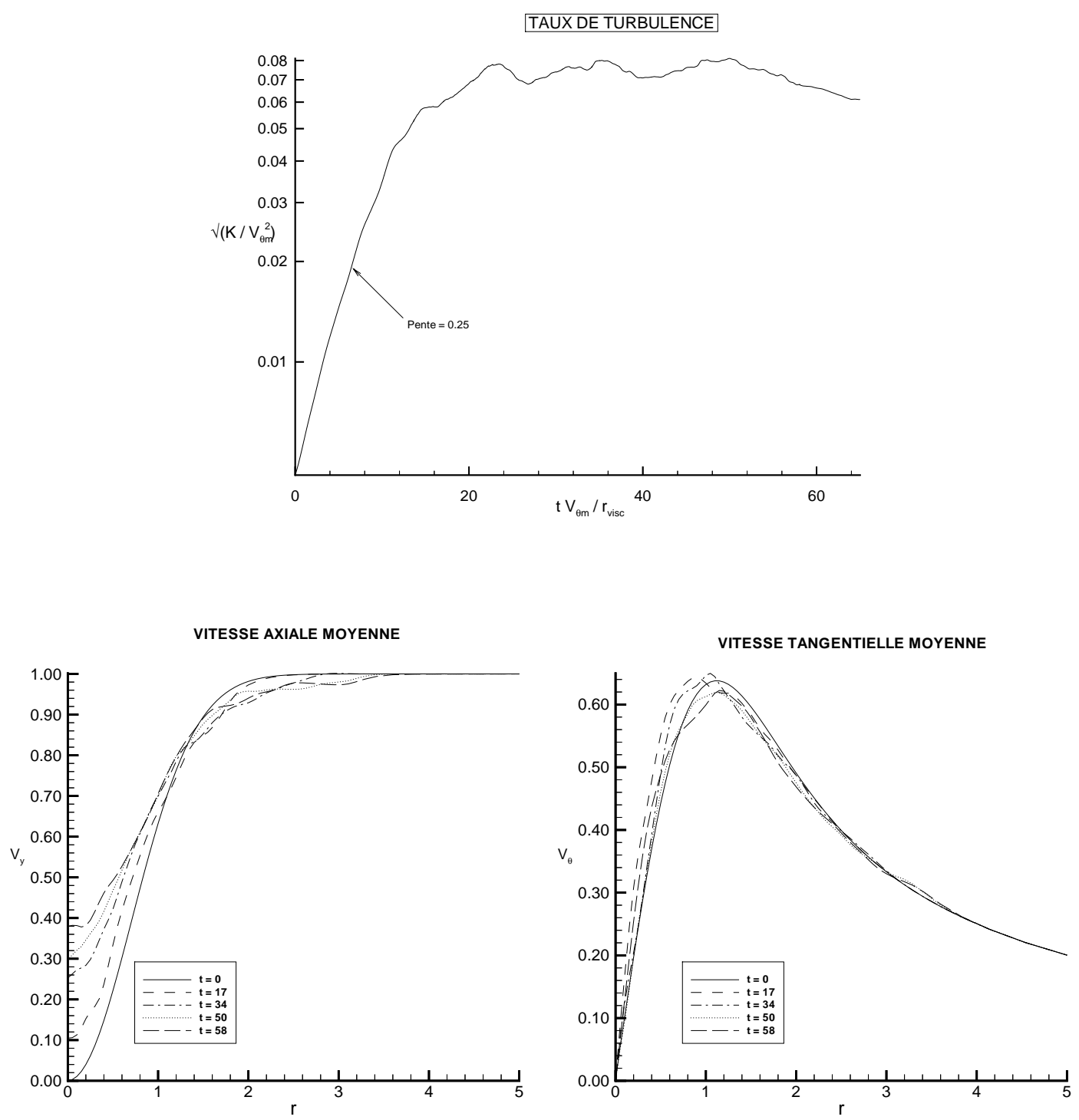

Figure 8: Upper plot : Turbulence level versus time. Lower left plot : Mean axial velocity versus the radius $r$. Lower right plot : Mean tangential velocity versus $r$. 